\title{
Bilateral ovarian micrometastatic adenocarcinoma upon prophylactic oophorectomy concurrent with low anterior resection for rectal cancer
}

\author{
Robin Irons* (D), Erin Mclntosh, Alexandre Hageboutros, David Warshal and Steven McClane
}

\begin{abstract}
Background: This case report draws attention to the debated role of prophylactic oophorectomy in women undergoing definitive surgical resection of colon and rectal cancers. It can be challenging to discern the indications and appropriate patient population for this procedure based on the current literature. Potential benefits include treatment and prevention of metastatic disease, preventing development of primary ovarian cancer, and prolonging survival. Negative effects include an increase in operative time and potential morbidity, development of osteoporosis, the risk of cardiac events, and decreasing sexual function. Multiple patient factors such as age, menopausal status, patient preference, presence of hereditary conditions, exposure to radiation, site, and stage of disease should be considered.
\end{abstract}

Case presentation: We present a case in which a premenopausal 49-year-old female underwent a prophylactic bilateral salpingo-oophorectomy concurrently with a low anterior resection following neoadjuvant chemoradiation for clinical stage III rectal cancer. On pathologic examination, resection margins and all 14 lymph nodes harvested were negative for malignancy. Interestingly, she was found to have micrometastatic adenocarcinoma in the bilateral ovaries which had appeared grossly normal at the time of surgery.

Conclusions: After consideration of the current literature, patient preference, and our clinical judgment, our patient ultimately had a therapeutic effect after undergoing prophylactic bilateral oophorectomy concurrently with a low anterior resection for rectal cancer. The addition of prophylactic oophorectomy in a select population, specifically women 50 years of age or younger and/or women who are in the premenopausal state, may carry a survival benefit in the setting of definitive surgical resection of colon and rectal cancers.

Keywords: Oophorectomy, Colorectal cancer, Prophylaxis, Metastasis, Ovarian cancer

\section{Background}

In this case report, we present a patient who underwent a prophylactic oophorectomy (PO) during a low anterior resection for clinical stage III rectal cancer after neoadjuvant chemotherapy and radiation who was found to have micrometastatic adenocarcinoma in the bilateral ovaries. The role of PO in primary colorectal cancers is controversial. Proposed benefits include decreasing

\footnotetext{
* Correspondence: irons-robin@cooperhealth.edu

Department of Surgery, Cooper University Hospital and Cooper Medical

School of Rowan University, Three Cooper Plaza, Suite 403, Camden, NJ 08103, USA
}

(c) The Author(s). 2017 Open Access This article is distributed under the terms of the Creative Commons Attribution 4.0 International License (http://creativecommons.org/licenses/by/4.0/), which permits unrestricted use, distribution, and reproduction in any medium, provided you give appropriate credit to the original author(s) and the source, provide a link to the Creative Commons license, and indicate if changes were made. The Creative Commons Public Domain Dedication waiver (http://creativecommons.org/publicdomain/zero/1.0/) applies to the data made available in this article, unless otherwise stated. ovarian cancer, and prolonging survival; however, there is limited data to support these proposals [1-3]. While the risk of immediate complications from the procedure are minimal, patients may experience long-term negative consequences including decreased sexual function, development of osteoporosis, and increased risk of cardiac events [4]. These negative consequences are largely offset by the use of exogenous estrogen therapy. The balance of the risks and benefits are affected by multiple patient factors including patient preference, age, menopausal 
status, presence of hereditary conditions, site, and stage of disease.

\section{Case presentation}

Our patient is a 49 -year-old, previously healthy, premenopausal female who presented to an outside institution's emergency department with a chief complaint of new onset, left lower quadrant pain and several weeks of constipation. She underwent her first colonoscopic evaluation which demonstrated a near obstructing mass at the rectosigmoid junction. She was transferred to our institution for comprehensive cancer care.

On admission interview, she reported that her obstructive symptoms had been progressively escalating, with her last bowel movement over a week ago. She denied personal history of cancer. Her family history was significant for a grandmother with gastric cancer in her late 60s. Pathology identified the mass as a moderately differentiated, invasive adenocarcinoma. She underwent a computed tomography (CT) scan of the chest, abdomen, and pelvis for staging. Imaging revealed wall thickening at the rectosigmoid junction and luminal narrowing (Fig. 1). Suspicious perirectal lymph nodes were present, but there was no evidence of distant metastasis (Fig. 2). Due to her progressive obstructive symptoms and abdominal pain, she was taken to the operating room for a diverting loop ileostomy and a rigid sigmoidoscopy which demonstrated the distal border of the lesion at approximately $9 \mathrm{~cm}$ from the anal verge.

During her initial admission, she was evaluated by our hematology/oncology and our radiation oncology department for outpatient neoadjuvant therapy. She underwent an MRI of the pelvis to further assist in staging which displayed a $4.6 \mathrm{~cm}$ mass located in the upper rectum that extended into the muscularis propria, classifying it as a T3b lesion. Enlarged superior rectal lymph nodes suspicious for malignancy were also noted (Fig. 3). Given her clinical diagnosis of stage III adenocarcinoma

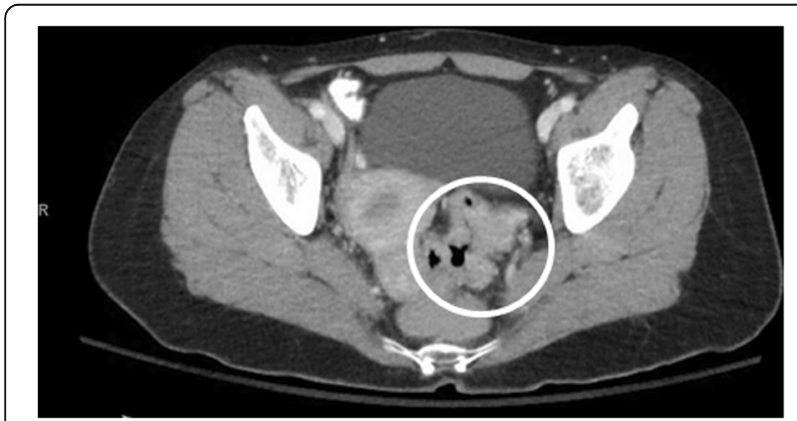

Fig. 1 Preoperative CT scan demonstrating a focal area of irregular wall thickening and associated luminal narrowing at the level of the rectosigmoid junction

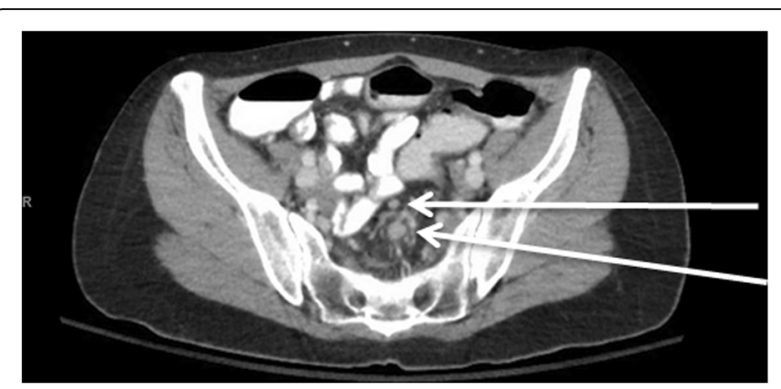

Fig. 2 Preoperative CT scan demonstrating multiple, borderline, perirectal lymph nodes with the largest measuring $1.0 \mathrm{~cm}$

of the rectum, she was deemed a candidate for chemotherapy with 5 -fluorouracil continuous infusion and concurrent radiation followed by definitive surgical resection.

Ten weeks after completion of preoperative chemoradiation, she underwent a repeat CT scan (Fig. 4). She had an appropriate response as evident by decreased rectal wall thickening and resolution of perirectal lymph node enlargement to the extent that nodes were no longer visualized.

She then underwent a laparoscopic low anterior resection. Our gynecology team was consulted prior to the procedure for a hysterectomy, as the patient was known to have a $4 \mathrm{~cm}$ posterior leiomyoma. Given her age, family history, recent chemotherapy, and pelvic radiation, the gynecologist felt she would benefit from a bilateral salpingo-oophorectomy which she had also consented for. On gross inspection, both ovaries appeared normal.

Pathology revealed moderately differentiated, ulcerated adenocarcinoma of the rectum with a depth of invasion

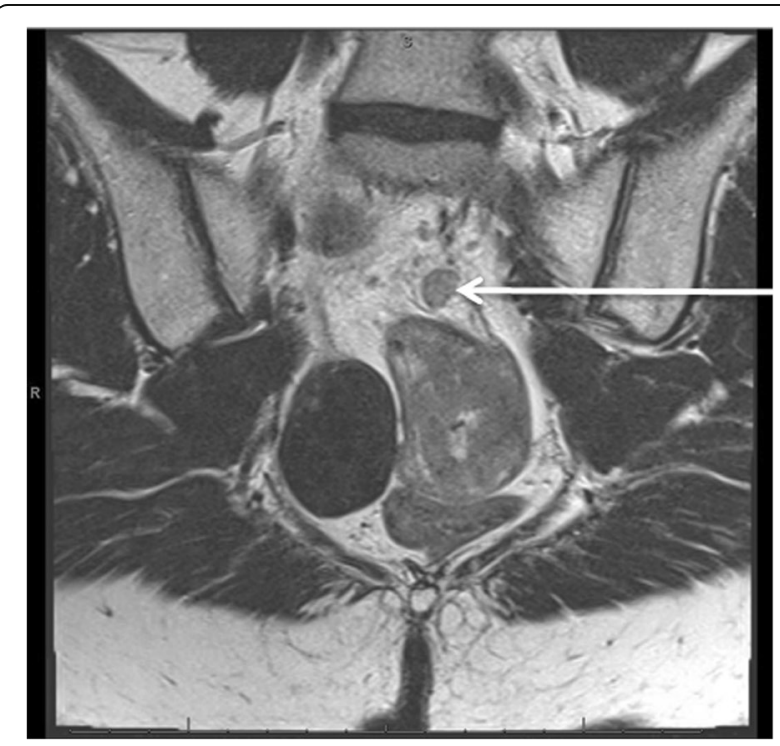

Fig. 3 Preoperative MRI demonstrating the largest $(0.9 \mathrm{~cm})$ of multiple perirectal lymph nodes 


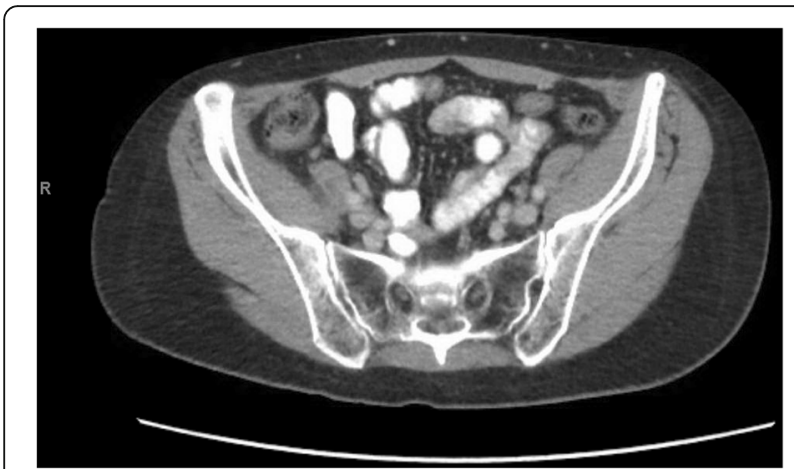

Fig. 4 Post neoadjuvant therapy CT scan demonstrating resolution of perirectal lymph node enlargement

to $0.1 \mathrm{~mm}$ from the peritoneal surface (Fig. 5). Proximal, distal, and radial resection margins were clear of malignancy and 0 of the 14 nodes harvested contained malignancy. Pericolorectal adipose contained tumor deposits and there was focal extramural vascular invasion. A $3.7 \mathrm{~cm}$ subserosal leiomyoma was present on the posterior aspect of the uterus which was consistent with gross examination. Of greater interest was the presence of metastatic rectal adenocarcinoma in the cortex of the left and right ovaries. Immunohistochemical testing of the rectal tumor for mismatch repair genes, $M S H 2$, MLH1, MSH6, and PMS2, associated with Lynch syndrome, was not suggestive of the disease.

\section{Discussion}

In women undergoing colorectal cancer resection, concurrent PO is not generally recommended or performed, yet it does need to be considered. These women carry a risk of developing metastatic disease to the ovaries in

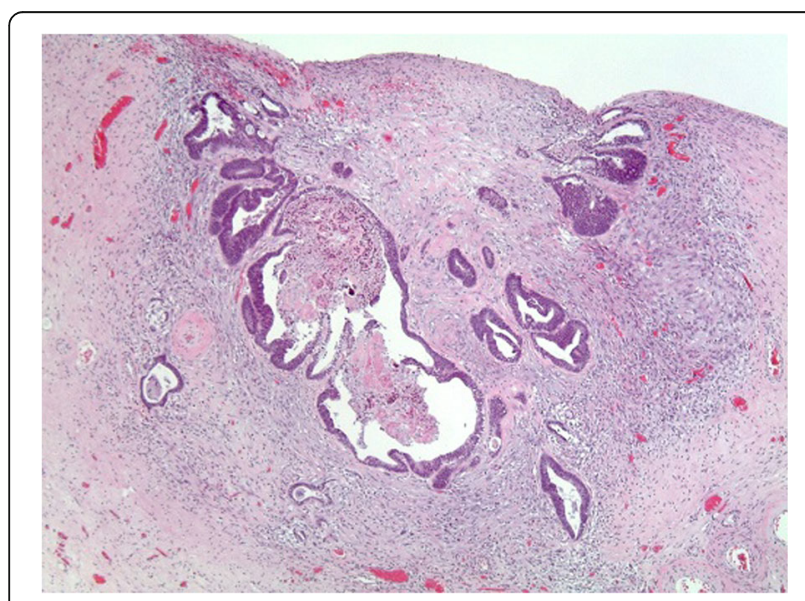

Fig. $5 \times 50$ H\&E stain. A small (up to $2.5 \mathrm{~mm}$ ) focus of adenocarcinoma is present in the cortex near the surface of the right ovary addition to their baseline risk of developing primary ovarian cancer. PO concurrently with definitive bowel resection has been proposed, as the ovaries are easily accessible via laparoscopy or laparotomy and resection carries minimal surgical risks. Current literature provides some insight into the risks women face with ovarian preservation, but guidelines for $\mathrm{PO}$ in this population have not been established.

\section{Metastasis disease to the ovaries}

The rate of metastasis of colorectal cancer to the ovaries in an average woman ranges from 1.4 to $4 \%[1,5]$. This rate significantly increases when evaluating women prior to menopause or at age 40 years or less [1, 6, 7]. Mackeigan et al. reviewed the clinical course of 484 women with colorectal cancer seen at Ferguson-Droste-Ferguson Hospital over an 8-year period. One hundred thirty-seven underwent oophorectomy with 133 prophylactically performed for grossly normal or suspicious involvement [7]. Eight (6\%) of the women were found to have metastatic disease to the ovaries. Premenopausal status was found to be associated with a higher rate of metastatic disease to the ovaries with $22 \%$ of premenopausal women affected.

Walton et al. reviewed 37 women with colorectal cancer who were less than 40 years old. Metastasis to the ovaries was noted in 5 (13.2\%) of these women. Recalde et al. identified 18 females diagnosed with colorectal cancer at age 35 or younger. Four (22\%) developed metastatic disease to the ovaries [5]. Blamey identified 882 women who underwent resection of a primary colorectal cancer. Thirteen (1.4\%) later required oophorectomy for ovarian recurrence [1]. The mean age of those who developed metastases to the ovaries was 51 years compared to 59 years in those who did not, which met a statistical significance. The lower incidence of ovarian recurrence in this study may be related to imperfections in long-term patient follow-up; therefore, the true incidence is not known.

\section{Incidence of primary ovarian cancer}

According to the 2010-2012 Surveillance, Epidemiology, and End Results Program, the average woman in the USA has a $1.3 \%$ chance of developing a primary ovarian cancer in their lifetime with a 5-year survival rate of $46.2 \%$. Age is a significant risk factor for the development of primary ovarian cancer with the median age of diagnosis being 63 years and $88 \%$ being diagnosed after the age of 45 [8]. Other contributing factors include but are not limited to obesity, reproductive history, use of hormone replacement therapy, and environmental factors [9].

McCredie et al. sought to establish the risk of developing a new primary cancer among those with a history of colorectal cancer using 20 years of data from the New South Wales Central Cancer Registry [3]. Patients with a 
diagnosis of invasive cancer of the colon or rectum who had survived beyond 2 months were identified. Patients who were diagnosed with a second primary within the first 2 months were omitted as they were thought to have two synchronous cancers. Compared to the general female population, women with colon cancer were found to be 2.8 times more likely to develop a primary ovarian cancer while those with rectal cancer were only 1.1 times more likely. Hereditary predispositions, hormonal factors, and dietary factors may contribute to this increased risk [3].

While high-dose radiation is known to increase the risk of developing ovarian cancer, the effect of the lower doses of radiation, such as those used in neoadjuvant therapy for colorectal cancer, is unclear. Recently, SEER data was utilized to retrospectively review the incidence of ovarian cancer over a 10-year period in 46,404 patients with rectal or rectosigmoid cancer [10]. Twenty $(0.15 \%)$ of the 13,099 patients who were treated with beam radiation were diagnosed with ovarian cancer compared to $91(0.27 \%)$ of the 33,305 patients who did not receive radiation therapy. Authors found a $44 \%$ decreased risk of ovarian cancer in the group who received radiation compared to the non-irradiated group. Pitfalls of this study include its retrospective design, use of correlation among variables, lack of information on patients' exposure to chemotherapy, radon, and total background exposures.

Based on these studies, risk factors for the development of primary ovarian cancer appear to include a history of colon cancer. Rectal cancer has not been demonstrated to increase risk of primary ovarian cancer which may be secondary to a protective effect that might occur with low-dose radiation.

\section{Ovarian cancer in grossly normal ovaries}

While gross abnormalities of the ovary can be appreciated upon visual inspection and palpation, not all metastatic ovarian disease are grossly apparent. Burt reviewed 493 cases of colorectal cancer treated at Presbyterian Hospital in New York over a 14-year period and found 17 cases of metastatic adenocarcinoma to the ovaries [11]. Four (24\%) had grossly normal ovaries on intraoperative inspection. In the previously mentioned Mackeigan study, $50 \%$ of women found to have ovarian metastases at the time of surgery had grossly normal ovaries [7].

\section{Impact on survival}

Impact of PO on survival within this population has not been clearly established due to limited sample sizes. Survival benefits may be affected by multiple patient characteristics including age, presence of hereditary syndromes, and stage of disease. The Mayo Clinic conducted a prospective, randomized trial encompassing 149 patients with Dukes' stages B or C colorectal cancer [12]. Patients were randomized to either PO or no oophorectomy. Survival curves suggested a potential survival benefit between 2 and 3 years post intervention, but this was not found to be a statistically significant difference and it did not persist at 5 years post intervention.

A retrospective review at the Mayo Clinic did not show a statistically significant survival advantage for 75 women who underwent oophorectomy when compared to the 496 patients in the control group [13]. Of interest, when patients were placed in age-based subgroups, the 5 women who were less than 50 years of age and had undergone PO survived more than 5 years. Cutait et al. examined a group of 335 women who underwent surgical resection of their colorectal cancer [2]. Two hundred one patients were selected based on individual surgeon's preference and judgment to undergo $\mathrm{PO}$ at the time of their initial definitive bowel resection. Four patients were found to have ovarian metastasis at the time of surgery. Disease-free survival, overall survival, and recurrence were evaluated with greater than 5-year follow-up achieved in $93 \%$ of the study population. There was no difference found in survival or recurrence when patients were stratified by menopausal status.

\section{Conclusions}

Determining the role of PO among women with resectable colorectal cancer remains difficult due to the limited amount of statistically significant data and the multiple patient variables at play. While older women are at a higher risk of developing a primary ovarian cancer, Walton, Blamey, and Recalde showed that women of a younger age were at a higher risk of having metastatic ovarian disease [6]. Additionally, Mackeigan showed an association with premenopausal status [7]. Although an improvement in survival is suggested in the women under 50 years of age, determining the effects of $\mathrm{PO}$ on survival requires a larger collection of data.

Menopausal status, age, chemotherapy, and radiation exposure, reproductive goals along with perspective on hormone replacement therapy should also be taken into consideration when counseling a patient. While the oophorectomized state is associated with increased risk of osteoporosis, coronary artery disease, decreased cognitive, and sexual functions, hormone therapy may help lessen these risks [4].

Given the not insignificant occurrence of incidentally found metastatic colorectal cancer to the ovaries in women undergoing resection, in addition to the risk of developing a primary ovarian cancer in years following resection, should prophylactic oophorectomy be offered to all women at the time of colorectal surgery? But more relevant may be this question in younger women, either under 50 years of age or in the premenopausal state, 
particularly those with colon cancer or with rectal cancer not receiving radiation therapy. These women, as well as those with strong family histories of other cancers, should certainly be strongly encouraged to consider undergoing concomitant oophorectomy.

Our patient was less than 50 years of age at presentation and had met her reproductive goals. She was premenopausal and underwent chemotherapy and pelvic radiation as part of her adjuvant therapy which often results in loss of ovarian function. The purpose of her surgical resection was to eliminate the risks of developing a primary or metastatic ovarian cancer, understanding the immediate and long-term risks of the oophorectomized state. Fortunately for her, the removal of her grossly normal appearing ovaries eliminated the only metastatic source of disease recurrence and should by all means improve her survival.

\section{Abbreviations}

CT: Computed tomography; PO: Prophylactic oophorectomy

\section{Acknowledgements}

The authors would like to thank Dr. Miles McFarland for providing the pathology report and images for this case presentation.

\section{Funding}

No funding was provided.

\section{Availability of data and materials}

As a case report, all data generated or analyzed are included in this published article and its supplementary information files.

\section{Authors' contributions}

RI performed a comprehensive review of patient's clinical course. She performed a literature review looking critically at the available data, drafted and revised the manuscript, and approved the final version prior to submission. She has agreed to be accountable for all aspects of the work. EM aided in summarizing the case, performing a critical review of the available data and revising the article for intellectual content. She gave final approval and she takes public responsibility for the work's content. $\mathrm{AH}$ aided in patient care, clinical decision making, expert critique of literature, and revising the article for intellectual content. He gave final approval and takes public responsibility for the work's content. DW aided in patient care, clinical decision making, expert critique of literature, and revising article for intellectual content. He gave final approval and takes public responsibility for the work's content. SM aided in formulation of the project idea, expert critique of literature, delivery of patient care, clinical decision making, drafting, and revising article for intellectual content. He gave final approval and takes public responsibility for the work's content.

\section{Competing interests}

The authors declare that they have no competing interests.

\section{Consent for publication}

Written informed consent for publication of their clinical details and/or clinical images was obtained from the patient. A copy of the consent form is available for review by the editor of this journal.
Received: 3 September 2016 Accepted: 1 February 2017

Published online: 07 February 2017

\section{References}

1. Blamey SL, McDermott FT, Pihl E, Hughes ES. Resected ovarian recurrence from colorectal adenocarcinoma: a study of 13 cases. Dis Colon Rectum. 1981;24(4):272-5.

2. Cutait R, Lesser ML, Enker WE. Prophylactic oophorectomy in surgery for large-bowel cancer. Dis Colon Rectum. 1983;26(1):6-11.

3. McCredie M, Macfarlane GJ, Bell J, Coates M. Second primary cancers after cancers of the colon and rectum in New South Wales, Australia, 1972-1991. Cancer Epidemiol Biomarkers Prev. 1997;6:155-60.

4. Erekson EA, Martin D, Ratner E. Oophorectomy: the debate between ovarian conservation and elective oophorectomy. Menopause. 2013;20:110-4.

5. Recalde M, Holyoke ED, Elias EG. Carcinoma of the colon, rectum, and anal canal in young patients. Surgery, gynecology \& obstetrics. 1974;139(6):909.

6. Walton WW, Hagihara PF, Griffen WO. Colorectal adenocarcinoma in patients less than 40 years old. Dis Colon Rectum. 1976;19(6):529-34.

7. Mackeigan JM, Ferguson JA. Prophylactic oophorectomy and colorectal cancer in premenopausal patients. Dis Colon Rectum. 1979;22(6):401-5.

8. Howlader N, Noone AM, Krapcho M, Miller D, Bishop K, Altekruse SF, Kosary CL, Yu M, Ruhl J, Tatalovich Z, Mariotto A, Lewis DR, Chen HS, Feuer EJ, Cronin KA (eds). SEER Cancer Statistics Review, 1975-2013, National Cancer Institute. Bethesda. http://seer.cancer.gov/csr/1975 2013/, based on November 2015 SEER data submission, posted to the SEER web site, April 2016.

9. Sueblinvong T, Carney ME. Current understanding of risk factors for ovarian cancer. Current treatment options in oncology. 2009;10(1-2):67-81.

10. Lehrer S, Green S, Rosenzweig KE. Reduced ovarian cancer incidence in women exposed to low dose ionizing background radiation or radiation to the ovaries after treatment for breast cancer or rectosigmoid cancer. Asian Pac J Cancer Prev. 2016;17(6):2979-82.

11. Burt Con Amore V. Carcinoma of the ovaries secondary to cancer of the colon and rectum. Diseases of the Colon \& Rectum. 1960;3(4):352-7.

12. Young-Fadok TM, Wolff BG, Nivatvongs $S$, Metzger PP, Ilstrup DM Prophylactic oophorectomy in colorectal carcinoma: preliminary results of a randomised prospective trial. Dis Colon Rectum. 1998:41:277-85. doi:10. 1007/BF02237479

13. Ballantyne GH, Reigel MM, Wolff BG, Ilstrup DM. Oophorectomy and colon cancer. Impact on survival Ann Surg. 1985;202(2):209-14
Submit your next manuscript to BioMed Central and we will help you at every step:

- We accept pre-submission inquiries

- Our selector tool helps you to find the most relevant journal

- We provide round the clock customer support

- Convenient online submission

- Thorough peer review

- Inclusion in PubMed and all major indexing services

- Maximum visibility for your research

Submit your manuscript at www.biomedcentral.com/submit
) BioMed Central 